\title{
Magnetism of Ho and Er Atoms on Close-Packed Metal Surfaces
}

\author{
F. Donati, ${ }^{1}$ A. Singha, ${ }^{1}$ S. Stepanow, ${ }^{2}$ C. Wäckerlin, ${ }^{1}$ J. Dreiser, ${ }^{1,3}$ P. Gambardella, ${ }^{2}$ S. Rusponi, ${ }^{1}$ and H. Brune ${ }^{1}$ \\ ${ }^{1}$ Institute of Condensed Matter Physics (ICMP), École Polytechnique Fédérale de Lausanne (EPFL), Station 3, \\ CH-1015 Lausanne, Switzerland \\ ${ }^{2}$ Department of Materials, ETH Zürich, Hönggerbergring 64, CH-8093 Zürich, Switzerland \\ ${ }^{3}$ Swiss Light Source (SLS), Paul Scherrer Institute (PSI)CH-5232 Villigen PSI, Switzerland
}

(Received 8 August 2014; published 3 December 2014)

\begin{abstract}
We investigated the magnetic properties of individual Ho atoms adsorbed on the (111) surface of Pt, which have been recently claimed to display single ion magnetic behavior. By combining x-ray absorption spectroscopy and magnetic dichroism measurements with ligand field multiplet calculations, we reveal a ground state which is incompatible with long spin relaxation times, in disagreement with former findings. A comparative study of the ground state and magnetic anisotropy of Ho and $\mathrm{Er}$ on $\mathrm{Pt}(111)$ and $\mathrm{Cu}(111)$ emphasizes the different interaction of the $4 f$ orbitals with localized and delocalized substrate states. In particular, we find a striking rotation of the magnetization easy axis for Er, which changes from out of plane on $\mathrm{Pt}(111)$ to in plane on $\mathrm{Cu}(111)$.
\end{abstract}

DOI: 10.1103/PhysRevLett.113.237201

PACS numbers: 75.30.Gw, 32.10.Dk, 75.10.Dg, 78.70.Dm

Single atoms adsorbed on a solid surface represent a paradigm for investigating the ultimate size limit of a magnet [1-6]. For transition-metal (TM) atoms, the reduced coordination on surface sites results in the survival of a large orbital moment, for which the spin-orbit coupling (SOC) may create magnetic anisotropies of the order of ten $[1,7]$ or several tens of meV [6]. In such quantum systems, the magnetic anisotropy is conveniently defined as the zerofield splitting (ZFS), the energy difference between the magnetic ground and first excited state. However, a large ZFS is only a necessary but not a sufficient condition for magnetic bistability. The angular symmetry of the magnetic anisotropy created by the crystal field (CF) of the surface determines the symmetry of the quantum states and thereby their coupling by tunnel matrix elements. This enables or disables magnetization reversal by tunneling. Additional mechanisms limiting the spin relaxation times are the scattering with substrate electrons and the interaction between the electron and nuclear spin of the magnetic atom. A close to ideal cylindrical $\mathrm{CF}$ environment and a ZFS of $58 \mathrm{meV}$ have been reported for Co atoms on $\mathrm{MgO} / \operatorname{Ag}(100)$; however, the spin-relaxation times, albeit reaching a record for TM atoms, remained relatively short with a lower bound of $230 \mu \mathrm{s}$ [6].

A promising alternative to $3 d$ elements are the $4 f$ rareearth (RE) elements. Their SOC is generally larger than the $\mathrm{CF}$ and therefore the magnetic states are eigenstates of the total angular momentum $J$ and its $z$ component $J_{z}$. A further consequence of the large SOC is that the energy splitting of the quantum levels can be even larger than in TM. Single ion RE molecular magnets show ZFSs up to $54 \mathrm{meV}$ [8-12], together with open hysteresis loops and magnetic lifetimes up to seconds at $3 \mathrm{~K}$ in the absence of an external field [13]. In addition, the strong localization of the
$4 f$ states leads to less hybridization with the surface, which could increase the lifetimes of the spin states. Rare earth atoms on a surface are of interest as model single ion magnets since the $\mathrm{CF}$ in the hollow adsorption sites has a dominant axial component, akin to the lanthanide molecular magnets $[8,9,14]$. A recent scanning tunneling microscopy (STM) study of single Ho atoms on Pt(111) reported a telegraph signal in the spin-polarized tunnel current caused by spin relaxation with characteristic times up to $700 \mathrm{~s}$ at $0.7 \mathrm{~K}$ [5]. This property was attributed to the combination of time reversal and CF symmetry $\left(\mathrm{C}_{3 v}\right)$ with a $J_{z}= \pm 8$ ground state of the Ho atom. However, this ground state has not been determined experimentally.

Here we present $\mathrm{x}$-ray absorption spectroscopy (XAS) and magnetic circular dichroism (XMCD) measurements that determine the electronic ground state and magnetic anisotropy of Ho and Er atoms deposited on Pt(111) and $\mathrm{Cu}(111)$. These elements show the largest orbital and total angular moments among the lanthanide series. For $\mathrm{Ho} / \mathrm{Pt}(111)$ we find an intermediate $J_{z}$ ground state, in striking disagreement with Ref. [5]. None of the investigated systems shows magnetic hysteresis at $2.5 \mathrm{~K}$. The comparison of $\mathrm{Ho}$ and $\mathrm{Er}$ on two close-packed metal surfaces reveals that the ground state and magnetic anisotropy of RE adatoms are more strongly influenced by the interaction with the substrate orbitals than by the symmetry of the adsorption site. Whereas $\operatorname{Pt}(111)$ provides more localized and directional $5 d$ orbitals, the $4 s$ orbitals of $\mathrm{Cu}(111)$ are delocalized and isotropic. Ho atoms have an out-of-plane easy axis on both surfaces and the largest $J_{z}$ ground state is realized only on $\mathrm{Cu}$, although with a smaller ZFS. The effect of the substrate is even more striking for $\mathrm{Er}$ where the easy axis changes from out of plane on $\mathrm{Pt}(111)$ to in plane on $\mathrm{Cu}(111)$. 
The XAS and XMCD measurements were performed at the X-Treme beam line at the Swiss Light Source [15]. Spectra were recorded with circularly and linearly polarized light in the total electron yield (TEY) mode at the Ho and Er $M_{4,5}$ absorption edges, at $T=2.5 \mathrm{~K}$, and in magnetic fields up to $B=6.8 \mathrm{~T}$ parallel to the $\mathrm{x}$-ray beam [16]. XAS and XMCD spectra were obtained from the sum, respectively, difference of the two circular polarizations. Multiplet calculations were performed to fit the XAS and XMCD spectra and the magnetization curves to obtain information on the nature of the ground states of the rare-earth ions $[6,16]$. Holmium and erbium were deposited from high purity rods (99\%) with an $e$-beam evaporator onto Pt and $\mathrm{Cu}(111)$ single crystals held at $3.5 \mathrm{~K}$ and in a background pressure not exceeding $4 \times 10^{-11} \mathrm{mbar}$. The rods were degassed for more than $100 \mathrm{~h}$ prior to evaporation [16].

We consider first the case of Ho monomers on Pt(111) [5]. Figure 1(a) shows the XAS of 0.03 monolayers (ML) Ho for both normal $\left(0^{\circ}\right)$ and grazing $\left(55^{\circ}\right)$ incidence. The XAS line shape is characteristic of a $4 f^{10}$ occupation [21] and exhibits very small differences depending on the x-ray incidence angle. As expected for the late lanthanides $\left(4 f^{n}\right.$, $n>7$ ), the $M_{4}$ and $M_{5}$ edges display the same sign of the dichroism, which indicates a large orbital moment parallel to the electron spin; see Fig. 1(b). The magnetic spin and orbital moments obtained from sum rules [22,23] are given in Table I. Note that the related value of $\left\langle J_{z}\right\rangle$ is significantly lower than the $J_{z}= \pm 8$ ground state calculated in Ref. [5]. The normal incidence magnetization curve saturates for $|B|>1.5 \mathrm{~T}$, while the grazing incidence curve reaches almost saturation at the maximum available field, see Fig. 1(c), thus indicating an out-of-plane easy axis. The absence of hysteresis indicates spin relaxations with a time scale shorter than a few tens of seconds at $2.5 \mathrm{~K}$ [16].

To gain further insight into the electronic ground state of Ho, the XAS and XMCD spectra as well as the magnetization curves were simulated by multiplet calculations including an external magnetic field $B$ and considering a $C_{3 v}$-symmetrical CF of the form [16]:

$$
\mathcal{H}_{\text {crys }}=B_{0}^{2} \hat{O}_{0}^{2}+B_{0}^{4} \hat{O}_{0}^{4}+B_{3}^{4} \hat{O}_{3}^{4},
$$

where $B_{m}^{n}$ are the CF parameters for the Stevens operators $\hat{O}_{m}^{n}$ [24]. To limit the number of free parameters in the (a) $\mathrm{Ho} / \mathrm{Pt}(111)$ - XAS

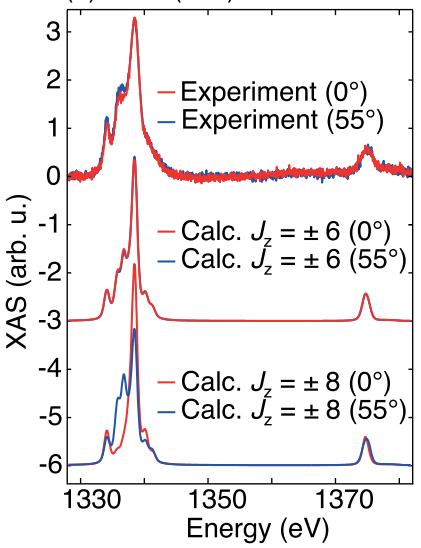

(c)

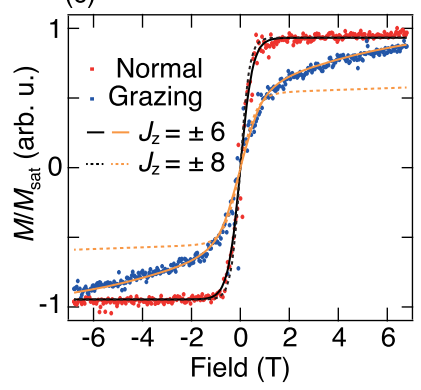

(b) $\mathrm{Ho} / \mathrm{Pt}(111)-\mathrm{XMCD}$
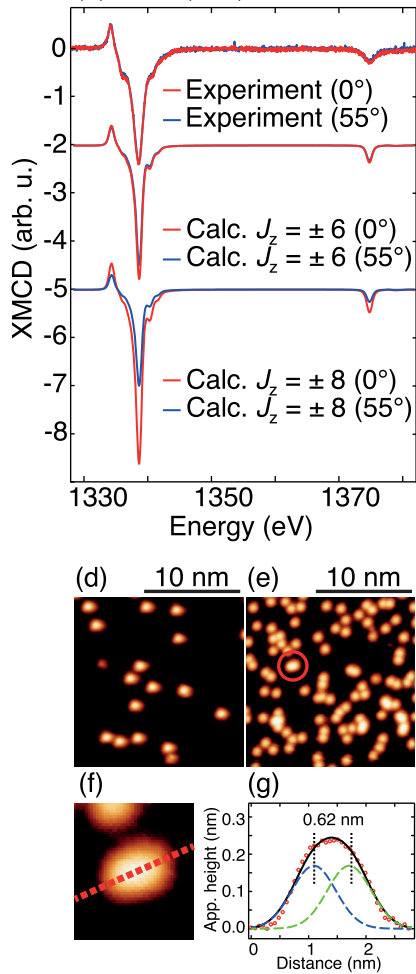

FIG. 1 (color online). Measured and simulated (a) XAS and (b) XMCD of $\operatorname{Ho} / \operatorname{Pt}(111)(\Theta=0.04 \mathrm{ML}, B=6.8 \mathrm{~T}$, $T=2.5 \mathrm{~K})$. Multiplet calculations are shown for a ground state doublet with $J_{\mathrm{z}}= \pm 6 \quad\left(B_{0}^{2}=-140 \mu \mathrm{eV}, B_{0}^{4}=1 \mu \mathrm{eV}\right.$, $\left.B_{3}^{4}=0 \mu \mathrm{eV}\right)$ and $J_{\mathrm{z}}= \pm 8$ (CF parameters from Ref. [5]). (c) Magnetization curves of $\mathrm{Ho} / \mathrm{Pt}(111)$ : measured (dots) and expectation values from multiplet calculations of $2\left\langle S_{\theta}(B)\right\rangle+$ $6\left\langle T_{\theta}(B)\right\rangle+\left\langle L_{\theta}(B)\right\rangle$ (solid and dashed lines). STM images of (d) 0.004 and (e) $0.02 \mathrm{ML} \mathrm{Ho} / \mathrm{Pt}(111)(T=4.3$ and $2.4 \mathrm{~K}$, respectively). (f) Magnification and (g) line profile of the apparent Ho dimer indicated in (e) (tunneling set point: $V_{t}=-50 \mathrm{mV}$, $I_{t}=100 \mathrm{pA}$ ). Red dots: experiments. The profile is fitted with two Gaussians using the apparent widths and heights of isolated atoms $(0.9 \mathrm{~nm}, 170 \pm 10 \mathrm{pm})$.

model, terms of higher order $B_{m}^{6} \hat{O}_{m}^{6}$ have been neglected. In addition, we do not include possible differences between the fcc- and hcp-adsorbed atoms, which are generally small due to the weak hybridization of the $4 f$ electrons [25]. The $\hat{O}_{0}^{2}$ and $\hat{O}_{0}^{4}$ operators contain only an even power of $\hat{J}_{z}$ and

TABLE I. Effective spin $\left(2\left\langle S_{\mathrm{z}}\right\rangle+6\left\langle T_{\mathrm{z}}\right\rangle\right)$, spin $\left(\left\langle S_{\mathrm{z}}\right\rangle\right)$, and orbital $\left(\left\langle L_{\mathrm{z}}\right\rangle\right)$ moments for Ho and Er atoms obtained applying sum rules to the normal incidence XMCD spectra assuming the number valence holes $n_{h}=4$ and 3, respectively. Values of $\left\langle S_{z}\right\rangle$ are obtained assuming that the experimental $\left\langle T_{z}\right\rangle /\left\langle S_{z}\right\rangle$ ratio is the same as that calculated within the multiplet model [16]. Values of $\left\langle J_{z}\right\rangle_{\text {calc }}$ at $B=6.8 \mathrm{~T}$ from multiplet calculations are shown for comparison, together with calculated ZFSs.

\begin{tabular}{|c|c|c|c|c|c|c|}
\hline & $2\left\langle S_{z}\right\rangle+6\left\langle T_{z}\right\rangle$ & $\left\langle S_{z}\right\rangle$ & $\left\langle L_{z}\right\rangle$ & $\left\langle J_{z}\right\rangle$ & $\left\langle J_{z}\right\rangle_{\text {calc }}$ & ZFS (meV) \\
\hline $\mathrm{Ho} / \mathrm{Pt}(111)$ & $2.84 \pm 0.13$ & $1.14 \pm 0.06$ & $4.28 \pm 0.06$ & $5.42 \pm 0.08$ & 6.00 & 3.9 \\
\hline $\mathrm{Ho} / \mathrm{Cu}(111)$ & $3.79 \pm 0.17$ & $1.52 \pm 0.07$ & $5.25 \pm 0.21$ & $6.77 \pm 0.22$ & 7.58 & 1.1 \\
\hline $\mathrm{Er} / \mathrm{Pt}(111)$ & $3.45 \pm 0.17$ & $1.04 \pm 0.05$ & $4.49 \pm 0.12$ & $5.53 \pm 0.13$ & 6.38 & 5.3 \\
\hline $\mathrm{Er} / \mathrm{Cu}(111)$ & $0.99 \pm 0.11$ & $0.31 \pm 0.03$ & $0.73 \pm 0.14$ & $1.04 \pm 0.14$ & 1.00 & 0.7 \\
\hline
\end{tabular}


their eigenfunctions are pure $J_{z}$ states. The transverse term $\hat{O}_{3}^{4}$ instead contains operators of the type $\hat{J}_{+}$and $\hat{J}_{-}$that mix states with different $J_{z}$. For $\mathrm{Ho} / \mathrm{Pt}(111)$, the spectra are very well reproduced using a purely uniaxial CF, see Figs. 1(a) and 1 (b), resulting in a $J_{z}= \pm 6$ ground state and a ZFS of $3.9 \mathrm{meV}$. Although the presented spectra were simulated with $B_{3}^{4}=0$, very similar results are obtained for $B_{3}^{4}$ values in a range of $\pm 5 \mu \mathrm{eV}$. Expectation values of the projected sum of the spin, dipolar, and orbital magnetic moments $2\left\langle S_{\theta}(B)\right\rangle+6\left\langle T_{\theta}(B)\right\rangle+\left\langle L_{\theta}(B)\right\rangle$ obtained from the multiplet calculations reproduce very well the experimental magnetization curves in Fig. 1(c), providing further evidence of a $J_{z}= \pm 6$ ground state. Calculations using the CF parameters given in Ref. [5] yield to a $J_{z}= \pm 8$ ground state with a strong angular variation of both XAS and XMCD in clear contrast to experiment; see Figs. 1(a) and 1(b). Moreover, the magnetization curves simulated for such a configuration do not reproduce the experimental curve at grazing incidence; see Fig. 1(c).

The remarkable differences between our results and those presented in Ref. [5] cannot be ascribed to the larger coverage employed to perform XMCD measurements. Figures 1(d) and 1(e) show two STM images, the first close to the coverage used in Ref. [5] and the latter close to the coverage used for our XMCD experiments. A statistical analysis of the Ho species based on their apparent heights reveals a large majority of isolated atoms for both coverages, $99 \pm 1 \%$ and $93 \pm 1 \%$ for 0.004 and 0.02 ML, respectively. The remaining species show an elongated shape and a larger apparent height, therefore suggesting that they are composed by two atoms in close proximity. A profile analysis over these apparent dimers reveals that the separation between the two constituent atoms is always larger than $0.55 \mathrm{~nm}$, corresponding to two or more lattice spacings of the Pt(111) surface; see Figs. 1(f) and 1(g). This indicates a short-range repulsion between Ho atoms preventing their adsorption onto nearest neighbor substrate sites. Atoms in close proximity on a $\operatorname{Pt}(111)$ surface were found to interact through substrate mediated RKKY interactions. For Co atoms, they have a characteristic energy of the order of $0.1 \mathrm{meV}$ [26] and a similar strength is expected for RE elements $[27,28]$, which is too small to play a role at the temperature and magnetic fields employed. The magnetic properties of the ensemble probed in XMCD are thus dominated by monomers, with the suppression of dimer formation further extending the coverage range where this is the case. Similarly, the slightly higher temperature employed in our experiments $(2.5 \mathrm{~K}$ in our work vs $0.7 \mathrm{~K}$ in Ref. [5]) may influence the spin relaxation time. However, the thermal energy at $2.5 \mathrm{~K}$ is $220 \mu \mathrm{eV}$, which is much smaller than the Ho/Pt(111) ZFS of $3.9 \mathrm{meV}$ and cannot affect the magnetic ground state. The discrepancy of our results with Ref. [5] therefore remains an open issue.

To explore the effect of a different electronic environment for the Ho atom, we performed a similar experiment

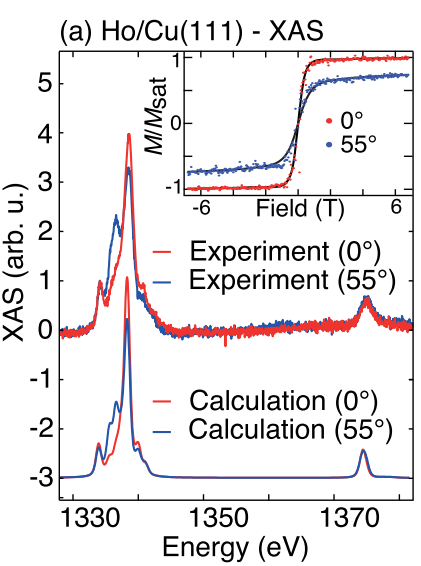

(b) $\mathrm{Ho} / \mathrm{Cu}(111)$ - XMCD

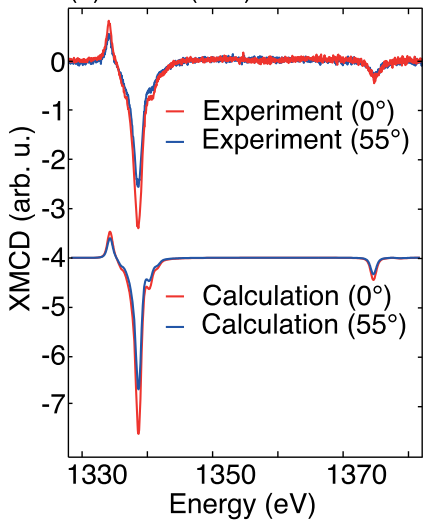

FIG. 2 (color online). Measured (a) XAS and (b) XMCD of $\mathrm{Ho} / \mathrm{Cu}(111)(\Theta=0.03 \mathrm{ML}, B=6.8 \mathrm{~T}, T=2.5 \mathrm{~K})$, together with simulated spectra $\left(B_{0}^{2}=-56 \mu \mathrm{eV}, B_{0}^{4}=0 \mu \mathrm{eV}, B_{3}^{4}=\right.$ $5.9 \mu \mathrm{eV}$ ). Inset: experimental (dots) and calculated values (lines) of $2\left\langle S_{\theta}(B)\right\rangle+6\left\langle T_{\theta}(B)\right\rangle+\left\langle L_{\theta}(B)\right\rangle$.

on $\mathrm{Cu}(111)$. Similarly to $\mathrm{Ho} / \mathrm{Pt}(111)$, the XAS spectra of Ho monomers on $\mathrm{Cu}(111)$ reveal a $4 f^{10}$ occupation; see Fig. 2(a). However, the differences between normal and grazing incidence are more pronounced. The dichroism is larger at normal incidence indicating an out-of-plane easy axis, as also visible from the magnetization curves. The magnetic moments from the sum rules, listed in Table I, indicate a ground state value of $\left\langle J_{z}\right\rangle=6.77$, which is larger than for $\mathrm{Ho} / \mathrm{Pt}(111)$. Multiplet calculations reproduce the experimental spectra and magnetization curves only by including a finite value of the transverse field parameter $B_{3}^{4}$; see Fig. 2. Therefore, the ground state is not a pure $J_{z}$ state; see Table I.

Figure 3 summarizes the energy splitting of the magnetic states of Ho atoms obtained from the CF parameters [16]. Note that the precision is higher on the low lying states due to the reduced parameter set employed to fit the experimental spectra and magnetization curves. The splitting is larger for Pt(111), with a ZFS of $3.9 \mathrm{meV}$. The $J_{z}= \pm 8$ states are higher in energy as a consequence of the $B_{0}^{4} \hat{O}_{0}^{4}$ term in $\mathcal{H}_{\text {crys. }}$. The resulting $J_{z}= \pm 6$ ground state is particularly delicate since it can be mixed by $\hat{O}_{3}^{4}$ operators, consequently allowing quantum tunneling of the magnetization. The mixing of the $J_{z}= \pm 6$ states thus produces a doublet with almost quenched $\left\langle J_{z}\right\rangle$; see Fig. 3. From our experimentally determined ground state, as well as from the energies and mixing of the quantum states, we do not expect large spin relaxation times for $\mathrm{Ho} / \mathrm{Pt}(111)$ at $B=0 \mathrm{~T}$, even at the slightly lower temperature and coverage of Ref. [5].

For $\mathrm{Ho} / \mathrm{Cu}(111)$, the ground state shows the largest $J_{z}$ among the magnetic states. Its value is reduced with respect to the free atom as a result of the transverse terms. The two ground states are not mixed by the transverse term; hence, quantum tunneling is suppressed at $B=0 \mathrm{~T}$, even if $B_{3}^{4}$ is 
(a) $\mathrm{Ho} / \mathrm{Pt}(111)$
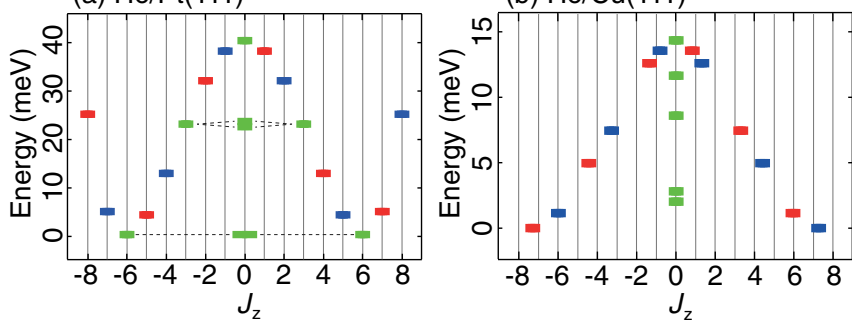

FIG. 3 (color online). Energy splitting of the quantum levels of a Ho atom on (a) $\mathrm{Pt}(111)$ and (b) $\mathrm{Cu}(111)$ obtained from the multiplet calculations. Subsets of magnetic states shown with the same colors are mixed by the transverse term $B_{3}^{4}$. Dashed lines in (a) depict the effect of $B_{3}^{4}=5 \mu \mathrm{eV}$ mixing the $J_{z}= \pm 3, \pm 6$ states to form singlets or doublets with almost quenched $\left\langle J_{z}\right\rangle$.

nonzero, as demonstrated in Ref. [5]. However, the weaker CF induces a ZFS of $1.1 \mathrm{meV}$, which is only 3 times higher than the thermal energy at $T=2.5 \mathrm{~K}$. Since each state of the first excited doublet is mixed with the ground state with opposite sign of $J_{z}$, the Ho magnetization can relatively easily switch from one ground state to the other via combined thermal excitation and electron scattering processes [5,29]. Therefore, a short lifetime of the magnetization is also expected for $\mathrm{Ho} / \mathrm{Cu}(111)$, as confirmed by the absence of hysteresis in the magnetization curves; see inset of Fig. 2(c).

We now turn to Er atoms deposited on $\mathrm{Pt}(111)$ and on $\mathrm{Cu}(111)$. For both systems, the XAS [Figs. 4(a) and 4(b)] is characteristic of a $4 f^{11}$ configuration [21]. Compared to Ho, Er atoms show a stronger dependence of XAS and dichroism on the incidence angle, see Figs. 4(a) and 4(b), and a larger sensitivity to the change in substrate. For $\mathrm{Er} / \mathrm{Pt}(111)$, the XMCD signal is stronger in the out-of-plane direction, while for $\mathrm{Er} / \mathrm{Cu}(111)$ the in-plane component becomes dominant. This difference is clearly reflected in the magnetization curves, which reveal a change of the easy axis from perpendicular to in plane [see Figs. 4(c) and 4(d)], and is correctly reproduced by our multiplet calculations [16]. The corresponding energy splitting of the magnetic levels is shown in the insets of Figs. 4(a) and 4(b). Similarly to the case of Ho, the $\mathrm{Pt}(111)$ surface provides a relatively strong $\mathrm{CF}$ for the Er atoms, generating a large energy splitting. Conversely, the $\mathrm{Cu}(111) \mathrm{CF}$ is weaker and induces a smaller splitting in the Er magnetic states, with the energy of the states increasing monotonically with $\left|J_{z}\right|[16]$.

The variety of magnetic behaviors observed combining Ho and Er atoms and (111) metal surfaces can be interpreted on the basis of the interaction between the surface LDOS and the $4 f$ charge distribution of the RE element. The spatial localization of the $4 f$ orbitals limits their hybridization with the substrate electrons, making the interaction with the surface almost purely electrostatic in nature $[14,30]$. This interaction induces a $4 f$ charge
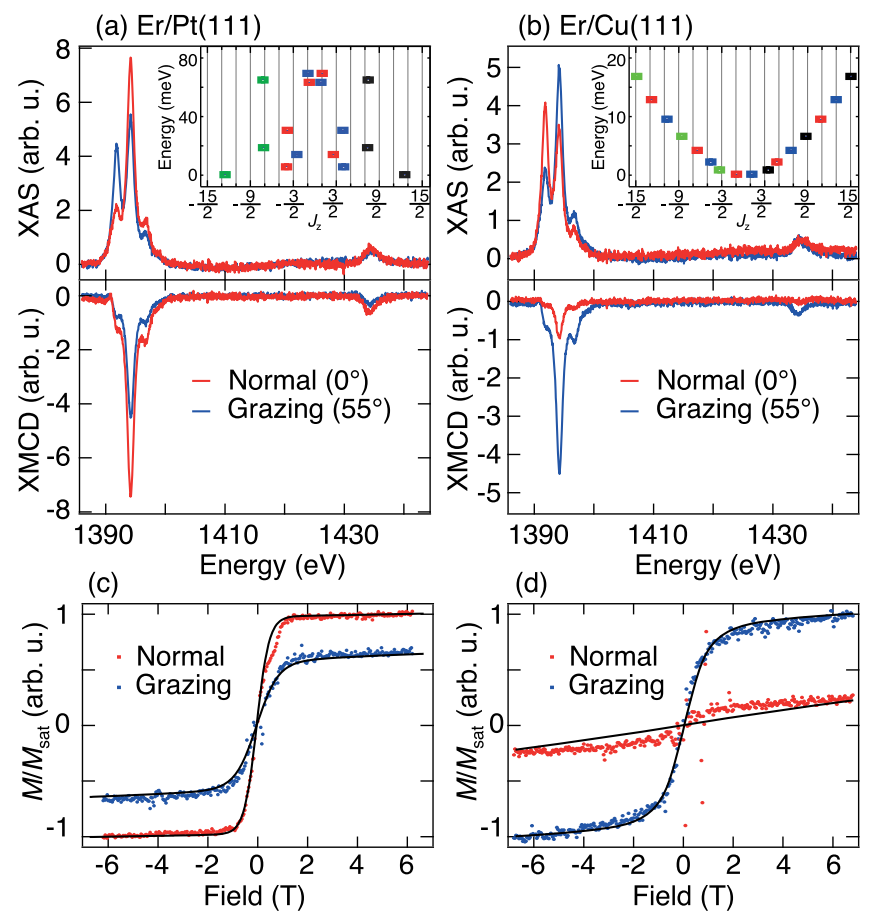

FIG. 4 (color online). Experimental XAS and XMCD of (a) $\operatorname{Er} / \operatorname{Pt}(111) \quad(\Theta=0.05 \mathrm{ML})$ and (b) $\operatorname{Er} / \mathrm{Cu}(111)$ $(\Theta=0.04 \mathrm{ML})$ measured at $B=6.8 \mathrm{~T}, T=2.5 \mathrm{~K}$. Insets: calculated energy splitting $\left[\operatorname{Er} / \operatorname{Pt}(111): B_{0}^{2}=-96 \mu \mathrm{eV}\right.$, $B_{0}^{4}=-0.85 \mu \mathrm{eV}, B_{3}^{4}=39.3 \mu \mathrm{eV} ; \mathrm{Er} / \mathrm{Cu}(111): B_{0}^{2}=96 \mu \mathrm{eV}$, $\left.B_{0}^{4}=0.0 \mu \mathrm{eV}, B_{3}^{4}=2.4 \mu \mathrm{eV}\right]$. Subsets of magnetic states shown with the same colors are mixed by the transverse term $B_{3}^{4}$. Magnetization curves of (c) $\operatorname{Er} / \operatorname{Pt}(111)$ and (d) $\mathrm{Er} / \mathrm{Cu}(111)$. Experimental (dots) and calculated (solid lines) expectation values of $2\left\langle S_{\theta}(B)\right\rangle+6\left\langle T_{\theta}(B)\right\rangle+\left\langle L_{\theta}(B)\right\rangle$.

distribution which can be very different from that of the corresponding free atom in the maximum $J_{z}$ state [Figs. 5(a) and 5(b)]. The $\mathrm{Cu}(111)$ LDOS is dominated by the delocalized $4 s$ states, which acts in first approximation
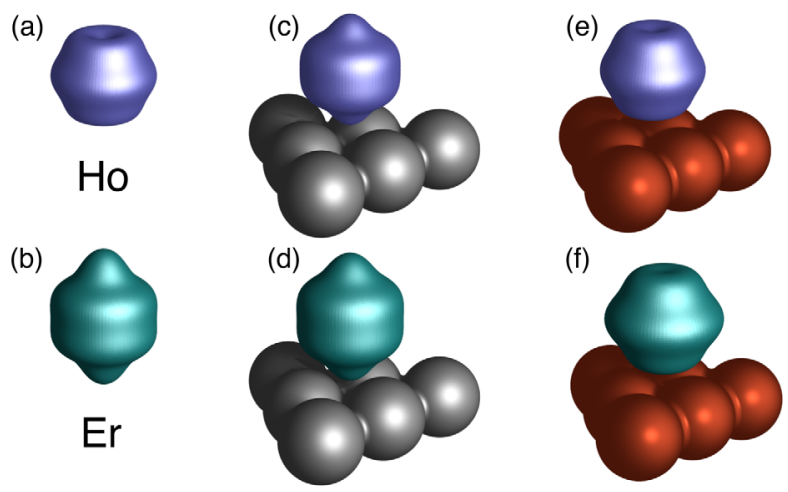

FIG. 5 (color online). Schematics of the $4 f$ charge distribution: (a),(b) free Ho and $\mathrm{Er}$ atoms, respectively, in the maximum $J_{z}$ state; ground states of the RE adatoms on (c),(d) $\mathrm{Pt}(111)$, and (e),(f) $\mathrm{Cu}(111)$ with the $4 f$ occupations obtained from the multiplet calculations [16]. 
as a uniform charge plane underneath the adsorbed RE atom, with small perturbations due to the $C_{3 v}$ symmetry of the adsorption site. Therefore, the interaction with the $4 f$ orbitals is minimal for the most oblate charge distributions, see Figs. 5(e) and 5(f), which correspond to the maximal and minimal $J_{z}$ for Ho and Er, respectively [14]. Conversely, the LDOS of $\operatorname{Pt}(111)$ is dominated by the $5 d$ contributions and the interaction with the RE atom is obtained through a more directional ligand field. The minimal repulsion is thus obtained minimizing the occupancy of the diagonal $4 f$ orbitals, which point towards the $\mathrm{Pt}$ atoms, thus forcing a prolate charge density distribution in the RE atoms, see Figs. 5(c) and 5(d), and ultimately determining the magnetic ground state.

In conclusion, our results shed light on the interaction of $\mathrm{RE}$ atoms with close-packed metal surfaces. More important than the symmetry of the adsorption site, the $d$ or $s$ character of the substrate LDOS plays a major role in determining the hierarchy of the $J_{z}$ states and ZFS, thus leading to nontrivial magnetic behavior on different surfaces. In all the investigated cases, we do not find evidence of long spin relaxation times down to $2.5 \mathrm{~K}$.

We acknowledge funding from the Swiss National Science Foundation. J. D. gratefully acknowledges funding by an SNF Ambizione Grant (No. PZ00P2_142474).

[1] P. Gambardella, S. Rusponi, M. Veronese, S. S. Dhesi, C. Grazioli, A. Dallmeyer, I. Cabria, R. Zeller, P. H. Dederichs, K. Kern, C. Carbone, and H. Brune, Science 300, 1130 (2003).

[2] C. F. Hirjibehedin, C.-Y. Lin, A. F. Otte, M. Ternes, C. P. Lutz, B. A. Jones, and A. J. Heinrich, Science 317, 1199 (2007).

[3] H. Brune and P. Gambardella, Surf. Sci. 603, 1812 (2009).

[4] A. A. Khajetoorians, B. Baxevanis, C. Hübner, T. Schlenk, S. Krause, T. O. Wehling, S. Lounis, A. Lichtenstein, D. Pfannkuche, J. Wiebe, and R. Wiesendanger, Science 339, 55 (2013).

[5] T. Miyamachi, T. Schuh, T. Märkl, C. Bresch, T. Balashov, A. Stöhr, C. Karlewski, S. André, M. Marthaler, M. Hoffmann, M. Geilhufe, S. Ostanin, W. Hergert, I. Mertig, G. Schön, and W. Wulfhekel, Nature (London) 503, 242 (2013).

[6] I. G. Rau, S. Baumann, S. Rusponi, F. Donati, S. Stepanow, L. Gragnaniello, J. Dreiser, C. Piamonteze, F. Nolting, S. Gangopadhyay, O. R. Albertini, R. M. Macfarlane, C. P. Lutz, B. A. Jones, P. Gambardella, A. J. Heinrich, and H. Brune, Science 344, 988 (2014).

[7] F. Donati, Q. Dubout, G. Autès, F. Patthey, F. Calleja, P. Gambardella, O. V. Yazyev, and H. Brune, Phys. Rev. Lett. 111, 236801 (2013).
[8] N. Ishikawa, M. Sugita, T. Ishikawa, S.-y. Koshihara, and Y. Kaizu, J. Am. Chem. Soc. 125, 8694 (2003).

[9] N. Ishikawa, M. Sugita, and W. Wernsdorfer, J. Am. Chem. Soc. 127, 3650 (2005).

[10] M. A. AlDamen, J. M. Clemente-Juan, E. Coronado, C. Martí-Gastaldo, and A. Gaita-Ariño, J. Am. Chem. Soc. 130, 8874 (2008).

[11] R. Westerström, J. Dreiser, C. Piamonteze, M. Muntwiler, S. Weyeneth, H. Brune, S. Rusponi, F. Nolting, A. Popov, S. Yang, L. Dunsch, and T. Greber, J. Am. Chem. Soc. 134, 9840 (2012).

[12] J. Dreiser, C. Wäckerlin, M. E. Ali, C. Piamonteze, F. Donati, A. Singha, K. S. Pedersen, S. Rusponi, J. Bendix, P. M. Oppeneer, T. A. Jung, and H. Brune, ACS Nano 8, 4662 (2014).

[13] N. Ishikawa, M. Sugita, T. Ishikawa, S.-y. Koshihara, and Y. Kaizu, J. Phys. Chem. B 108, 11265 (2004).

[14] J. D. Rinehart and J. R. Long, Chem. Sci. 2, 2078 (2011).

[15] C. Piamonteze, U. Flechsig, S. Rusponi, J. Dreiser, J. Heidler, M. Schmidt, R. Wetter, M. Calvi, T. Schmidt, H. Pruchova, J. Krempasky, C. Quitmann, H. Brune, and F. Nolting, J. Synchrotron Radiat. 19, 661 (2012).

[16] See Supplemental Material at http://link.aps.org/ supplemental/10.1103/PhysRevLett.113.237201, which includes Refs. [17-20], for details concerning spectra acquisition, sample preparation, and multiplet calculations.

[17] F. de Groot, Coord. Chem. Rev. 249, 31 (2005).

[18] R. D. Cowan, The Theory of Atomic Structure and Spectral (University of California Press, Berkeley, 1981).

[19] F. de Groot and A. Kotani, Core Level Spectroscopy of Solids (CRC Press, Boca Raton, 2008).

[20] G. van der Laan and B. T. Thole, Phys. Rev. B 43, 13401 (1991).

[21] B. T. Thole, G. van der Laan, J. C. Fuggle, G. A. Sawatzky, R. C. Karnatak, and J.-M. Esteva, Phys. Rev. B 32, 5107 (1985).

[22] B. T. Thole, P. Carra, F. Sette, and G. van der Laan, Phys. Rev. Lett. 68, 1943 (1992).

[23] P. Carra, B. T. Thole, M. Altarelli, and X. Wang, Phys. Rev. Lett. 70, 694 (1993).

[24] A. Abragam and B. Bleaney, Electron Paramagnetic Resonance of Transition Ions (Clarendon Press, Oxford, 1970).

[25] T. Schuh, T. Miyamachi, S. Gerstl, M. Geilhufe, M. Hoffmann, S. Ostanin, W. Hergert, A. Ernst, and W. Wulfhekel, Nano Lett. 12, 4805 (2012).

[26] L. Zhou, J. Wiebe, S. Lounis, E. Vedmedenko, F. Meier, S. Blügel, P. H. Dederichs, and R. Wiesendanger, Nat. Phys. 6, 187 (2010).

[27] C.-Y. Lin, J.-L. Li, Y.-H. Hsieh, K.-L. Ou, and B. A. Jones, Phys. Rev. X 2, 021012 (2012).

[28] B. Bryant, A. Spinelli, J. J. T. Wagenaar, M. Gerrits, and A. F. Otte, Phys. Rev. Lett. 111, 127203 (2013).

[29] J. P. Gauyacq, N. Lorente, and F. D. Novaes, Prog. Surf. Sci. 87, 63 (2012).

[30] R. Skomski and D. Sellmyer, Journal of rare earths / Chinese Society of Rare Earths 27, 675 (2009). 


\title{
Magnetism of Ho and Er Atoms on Close-Packed Metal Surfaces
}

\author{
F. Donati, ${ }^{1}$ A. Singha,${ }^{1}$ S. Stepanow ${ }^{2}$ C. Wäckerlin, ${ }^{1}$ J. Dreiser,${ }^{1,3}$ P. Gambardella, ${ }^{2}$ S. Rusponi, ${ }^{1}$ and H. Brune ${ }^{1}$ \\ ${ }^{1}$ Institute of Condensed Matter Physics (ICMP), \\ École Polytechnique Fédérale de Lausanne (EPFL), Station 3, CH-1015, Switzerland \\ ${ }^{2}$ Department of Materials, ETH Zürich, Hönggerbergring 64, CH-8093 Zürich, Switzerland \\ ${ }^{3}$ Swiss Light Source (SLS), Paul Scherrer Institut (PSI), CH-5232 Villigen PSI, Switzerland
}

\section{EXPERIMENTAL DETAILS FOR XMCD MEASUREMENTS}

We performed XAS and XMCD measurements at the X-Treme beamline of the Swiss Light Source. XAS spectra were recorded with circularly polarized light in the total electron yield (TEY) mode at the Ho and Er $M_{4,5}$ absorption edges at $T=2.5 \mathrm{~K}$ and in magnetic fields up to $B=6.8 \mathrm{~T}$ parallel to the X-ray beam. The spectra were normalized to the intensity of the X-ray beam measured on a metallic grid placed upstream of the sample, and in addition to their pre-edge values at 1328 and $1385 \mathrm{eV}$ for $\mathrm{Ho}$ and $\mathrm{Er}$, respectively, in order to account for the different TEY efficiencies at normal $\left(0^{\circ}\right)$ and grazing $\left(55^{\circ}\right)$ incidence. To isolate the RE contribution from the background of the metal substrate, XAS signals of the clean surfaces in the energy range of the $\mathrm{Ho}$ (Er) $M_{4,5}$ edges were subtracted to the XAS spectra recorded after the deposition of RE atoms. Magnetization curves were acquired at a speed of $0.0125 \mathrm{~T} / \mathrm{s}$ measuring the maximum of the XMCD at the Ho and $\operatorname{Er} M_{5}$ edges, respectively, normalized by the corresponding value of the XAS background. The XMCD maxima are thus proportional to the total moments of a Ho (Er) atom, but are less accurate than the full sum rule analysis taking into account integrals over both $M_{4,5}$ edges. We therefore scaled the normal and grazing curves such that their ratio at $6.8 \mathrm{~T}$ is equal to the ratio of the corresponding $2\left\langle S_{\theta}(B)\right\rangle+6\left\langle T_{\theta}(B)\right\rangle+\left\langle L_{\theta}(B)\right\rangle$ values from sum rules. Each point of the magnetization curve requires about $4 \mathrm{~s}$ to be acquired. This sets the time resolution of the experiment.

Single crystals of $\mathrm{Pt}(111)$ and $\mathrm{Cu}(111)$ were prepared in situ by means of $\mathrm{Ar}^{+}$sputtering $\left(4 \mu \mathrm{A} / \mathrm{cm}^{2}, 300 \mathrm{~K}, 1200\right.$ $\mathrm{eV}, 30 \mathrm{mins})$ and annealing cycles $(1300 \mathrm{~K}, 1 \mathrm{~min})$ in the preparation chamber of the X-Treme end station. X-Treme end station. The surface quality was checked with an Omicron VT scanning tunneling microscope (STM) connected to the preparation chamber. Holmium and erbium atoms were deposited using an e-beam evaporator from high purity rods $(99.9 \%)$ on $\mathrm{Pt}$ and $\mathrm{Cu}$ single crystals held at $3.5 \mathrm{~K}$ and in a background pressure not exceeding $4 \times 10^{-11}$ mbar. The rods were degassed for more than 100 hours prior to evaporation. The Ho and Er coverages $(\Theta)$ were calibrated by mapping the covered area with STM at room temperature for close to half-monolayer coverage and this calibration was used in XAS by evaluating the integrated Ho and $\operatorname{Er} M_{4,5}$ signal for the same sample. One monolayer (ML) is defined as one rare earth atom per substrate atom. For samples prepared under identical conditions as those measured with XMCD, we determined the fraction of monomers and dimers in the ensemble with a home-built low temperature STM.

The extremely high reactivity of rare earth single atoms on a surface imposes stringent requirements in terms of vacuum conditions and measurement duration. The cryostat has a base pressure of $2 \times 10^{-11} \mathrm{mbar}$ and is equipped with a non-emitting getter with a pumping speed of $1000 \mathrm{l} / \mathrm{s}$ (model D 1000 Saes Getter) which is activated before starting the experiments. In order to limit the exposure of the sample to residual vacuum contaminants, spectra acquisition started right after the deposition of $\mathrm{Ho}$ and Er atoms and lasted no longer than 5 hours. During this interval, we checked that the XAS and XMCD intensities varied by less than $5 \%$. Then, a new sample was freshly re-prepared.

\section{DETAILS FOR MULTIPLET CALCULATIONS}

The XAS simulations are based on an atomic multiplet model that takes into account the electron-electron interaction among $f$ - and $d$-electrons using rescaled Slater-Condon integrals, and the atomic spin-orbit interaction [17-20]. The finite overlap of the rare-earth ion wavefunctions with the surrounding atoms is neglected due to the well-screened character of the $4 f$-states. The x-ray absorption spectrum for a polarization vector $\boldsymbol{e}_{q}$ is calculated based on Fermi's golden rule considering only electric dipole allowed transitions,

$$
W_{f i} \propto\left|\left\langle\Psi_{f}\left|\boldsymbol{e}_{q} \cdot r\right| \Psi_{i}\right\rangle\right|^{2} \delta\left(E_{f}-E_{i}-\hbar \omega\right) .
$$


For the evaluation of the transition matrix elements the electric dipole operator for different polarizations and incidence angles is expressed by a linear combination of the spherical harmonics $Y_{1}^{0}, Y_{1}^{1}$, and $Y_{1}^{-1}$ which correspond to the electric field parallel to $z$ and the circular polarizations, respectively. The delta function assures energy conservation but will be replaced by a Lorentzian to account for the final lifetime of the core hole. The spectrum is further broadened by a Gaussian function to account for the experimental energy resolution. Due to the strong overlap of the core hole with the valence electron wavefunctions the calculations must take into account the interaction between the hole and the valence levels. Hence, multiplet effects dominate the spectral shape. At finite temperature, the population of excited states of the initial state configuration is also taken into account by considering transitions from Boltzmann weighted initial states. The Hamiltonian of the initial and final states, i.e., $3 d^{10} 4 f^{N}$ and $3 d^{9} 4 f^{N+1}$ for the $M$-edge, respectively, are diagonalized separately. The spectrum is then calculated from the sum of all possible transitions for an electron excited from the occupied $3 d$ level into an unoccupied $4 f$ level. In the crystal field limit, the ground state is given by a single electronic configuration $f^{N}$ (where $\mathrm{N}$ is the number of valence $f$-electrons), split in energy by electron repulsion and a crystal field potential with a certain symmetry. The atomic Hamiltonian is given by

$$
\begin{aligned}
H & =H_{\text {atom }}+H_{\mathrm{cf}}, \quad \text { where } \\
H_{\text {atom }} & =\sum_{N} \frac{p_{i}^{2}}{2 m}+\sum_{N} \frac{-Z e^{2}}{r_{i}}+\sum_{\text {pairs }} \frac{e^{2}}{r_{i j}}+\sum_{N} \zeta\left(r_{i}\right) l_{i} \cdot s_{i}, \quad \text { and } \\
H_{\mathrm{cf}} & =-e \sum_{N} V\left(\boldsymbol{r}_{i}\right) .
\end{aligned}
$$

This approach includes both electronic Coulomb interactions and spin-orbit coupling for each subshell. The first two terms of the atomic Hamiltonian $H_{\text {atom }}$ give the average energy of the configuration and contains the kinetic energy and the interaction of the electron with the nucleus. The third term is the electron-electron interactions term that results in the splitting of the electronic states into the well-known multiplets. The spherical part of the electron-electron repulsion can be separated and is added to the average energy. The remaining non-spherical part is treated explicitly. The last term in $H_{\text {atom }}$ represents the spin-orbit interaction that is calculated for each shell and $\zeta$ is assumed to be constant within a shell. Thus, the non-spherical part of the electron-electron interaction and the spin-orbit interaction determine the relative energies of the different states within the atomic configuration. The electronic environment of the ion is taken into account by the crystal field Hamiltonian $H_{\mathrm{cf}}$,

$$
V(r, \theta, \phi)=\sum_{n=0}^{\infty} \sum_{m=0}^{n} r^{n} \gamma_{n m} Z_{n m}(\theta, \phi),
$$

where

$$
\gamma_{n m}=\sum_{j=1}^{k} \frac{4 \pi}{2 n+1} q_{j} \frac{Z_{n m}\left(\theta_{j}, \phi_{j}\right)}{r_{j}^{n+1}}
$$

$k$ are the number of coordinating atoms with charge $q_{j}$ at positions $\left(r_{j}, \theta_{j}, \phi_{j}\right)$. The $Z_{n m}(\theta, \phi)$ are the tesseral harmonics and the parameters $\gamma_{n m}$ can be calculated from the positions and charges of the surrounding atoms. In general the potential function reflects the point symmetry of the lattice site. The less symmetric the site, the more terms occur in the expansion. It must be noted that the terms occurring depend on the axes chosen, and are in their simplest form when the axes are the symmetry axes of the point group. There are two general rules; Firstly, if the lattice site posses inversion symmetry all odd- $n$ terms vanish, and secondly, if the $z$ axis is an $m$-fold axis of symmetry, the potential will contain terms of $Z_{n m}$. For some high symmetry cases there exist relations between the coefficients of the tesseral harmonics of the same $n$. Nevertheless, not all the nonzero terms in the expansion of the potential will affect the energy levels of the ion, as their matrix elements may yet be zero. More specifically, for $f$-shell electrons only $n \leq 6$ terms yield non-zero matrix elements. Hence, alternatively instead of calculating the non-zero $\gamma_{n m}$ parameters the prefactors $A_{n m}=\left\langle r^{n}\right\rangle \gamma_{n m}$ are treated as fit parameters in the calculations to match the experimental lineshape. In model Hamiltonian acting mostly only on the ground state multiplet the crystal field potential is usually expressed in terms of the Stevens operators $\hat{O}_{m}^{n}$,

$$
V_{\mathrm{cf}}=\sum_{n, m} B_{m}^{n} \hat{O}_{m}^{n}
$$


Note, that there exist a direct relationship between the $A_{n m}$ and $B_{m}^{n}$ parameters that depends on the number of electrons in the considered shell. Thus the rules for non-vanishing $\gamma_{n m}$ values aply also to the $B_{m}^{n}$ parameters. The crystal field term is only applied to the outer shell, since the core hole in the excited state is well screened by the other electrons. In addition the crystal field applied to the initial and final state is chosen to be the same, although the presence of the core hole and the Coulomb repulsion of the excited electron affects the radial extension of the wavefunction and hence the crystal field term. We neglect this effect in the XAS calculations. The spectral shape is mostly determined by the final state crystal field term and the nature of the ground state.

To calculate XAS spectra of magnetic ions and hence XMCD spectra, the Zeeman energy term,

$$
H_{\mathrm{Z}}=\sum_{i=1}^{N} \frac{\mu_{\mathrm{B}}}{\hbar} \boldsymbol{B} \cdot\left(2 \boldsymbol{s}^{i}+\boldsymbol{l}^{i}\right)
$$

is added to the initial and final state Hamiltonian acting only on the valence shell. Here, $\boldsymbol{s}^{i}$ and $\boldsymbol{l}^{i}$ are the one-electron spin and orbital kinetic momentum operators that add up to give the total atomic spin $(\boldsymbol{S})$ and orbital moments $(\boldsymbol{L})$, respectively.

The many electron wavefunction of a single configuration is represented by a linear combination of determinantal product states with basis wavefunctions of the form $R_{n}(r) Y_{m}^{k}(\theta, \phi) \chi(\sigma)$, which separates into the radial part $R_{n}(r)$, the spherical harmonics $Y_{m}^{k}$ for the angular dependence, and the spin function $\chi(\sigma)$. The matrix elements of the radial part for the different terms of the Hamiltonian are expressed by the Slater-Condon-Shortley parameters $\left(F_{i}\right.$ and $\left.G_{i}\right)$. These values have been reduced to 73 percent of their Hartree-Fock calculated values to account for the overestimation of electron-electron repulsion in the free ion and for the delocalization and screening effects in the adatom system. The spin-orbit coupling constant $\zeta$ is obtained together with the $F_{i}$ and $G_{i}$ values using the atomic theory code developed by Cowan [18]. The non-spherical part of the total Hamiltonian for the initial and final state is numerically diagonalized considering all contributions (electron-electron interaction, ligand field, spin-orbit coupling and magnetic field) simultaneously. This yields wavefunctions and energies from which we calculate also the expectation values of the spin and orbital moments. Our code is free of symmetry restrictions, i.e., external fields can be applied in any possible direction.

The expectation values of the magnetic moments for the eigenstates can be directly calculated. The total moment of a shell is given by the operator

$$
M=\sum_{N} m_{i}
$$

where the $m_{i}$ are the single electron spin or angular momentum operators. The same applies for the spin dipole operator. The expectation value of moment $\left\langle M_{\theta}^{i}\right\rangle$ of state $i$ along the direction $\boldsymbol{e}_{\theta}$ is given by

$$
\begin{aligned}
\left\langle M_{\theta}^{i}\right\rangle & =\left\langle\Psi_{i}\left|M_{\theta}\right| \Psi_{i}\right\rangle \\
& =\left(\boldsymbol{e}_{\theta} \cdot \boldsymbol{e}_{x}\right)\left\langle M_{x}^{i}\right\rangle+\left(\boldsymbol{e}_{\theta} \cdot \boldsymbol{e}_{y}\right)\left\langle M_{y}^{i}\right\rangle+\left(\boldsymbol{e}_{\theta} \cdot \boldsymbol{e}_{z}\right)\left\langle M_{z}^{i}\right\rangle .
\end{aligned}
$$

To calculate the expectation value of the Moment $M$ at finite temperature, the states are weighted by the Boltzmann distribution according to

$$
\begin{aligned}
\left\langle M_{\theta}(T)\right\rangle & =\frac{1}{Z} \sum_{i}\left\langle M_{\theta}^{i}\right\rangle e^{-\frac{E_{i}}{k_{\mathrm{B}} T}} \text { with } \\
Z & =\sum_{i} e^{-\frac{E_{i}}{k_{\mathrm{B}} T}} .
\end{aligned}
$$

III. CRYSTAL FIELD PARAMETERS FOR MULTIPLET CALCULATIONS 


\begin{tabular}{l|cccccc} 
System & $A_{2,0}$ & $A_{4,0}$ & $A_{4,3}$ & $B_{0}^{2}$ & $B_{0}^{4}$ & $B_{3}^{4}$ \\
\hline \hline $\mathrm{Ho} / \mathrm{Pt}(111)$ & $+0.20 \mathrm{eV}$ & $-0.30 \mathrm{eV}$ & $+0.00 \mathrm{eV}$ & $-140 \mu \mathrm{eV}$ & $+1.06 \mu \mathrm{eV}$ & $+0.0 \mu \mathrm{eV}$ \\
$\mathrm{Ho} / \mathrm{Cu}(111)$ & $+0.08 \mathrm{eV}$ & $+0.00 \mathrm{eV}$ & $-0.10 \mathrm{eV}$ & $-56 \mu \mathrm{eV}$ & $+0.00 \mu \mathrm{eV}$ & $+5.9 \mu \mathrm{eV}$ \\
$\mathrm{Er} / \mathrm{Pt}(111)$ & $-0.12 \mathrm{eV}$ & $-0.18 \mathrm{eV}$ & $+0.50 \mathrm{eV}$ & $-96 \mu \mathrm{eV}$ & $-0.85 \mu \mathrm{eV}$ & $+39.3 \mu \mathrm{eV}$ \\
$\mathrm{Er} / \mathrm{Cu}(111)$ & $+0.12 \mathrm{eV}$ & $+0.00 \mathrm{eV}$ & $+0.03 \mathrm{eV}$ & $+96 \mu \mathrm{eV}$ & $+0.00 \mu \mathrm{eV}$ & $+2.4 \mu \mathrm{eV}$ \\
\hline \hline
\end{tabular}

Table I. Crystal field parameters $A_{n m}$ employed in the multiplet calculations, together with the corresponding $B_{m}^{n}$ terms used in Eq. 1 of the main text.

IV. XAS MULTIPLET SIMULATIONS FOR ER

(a) $\operatorname{Er} / \operatorname{Pt}(111)$

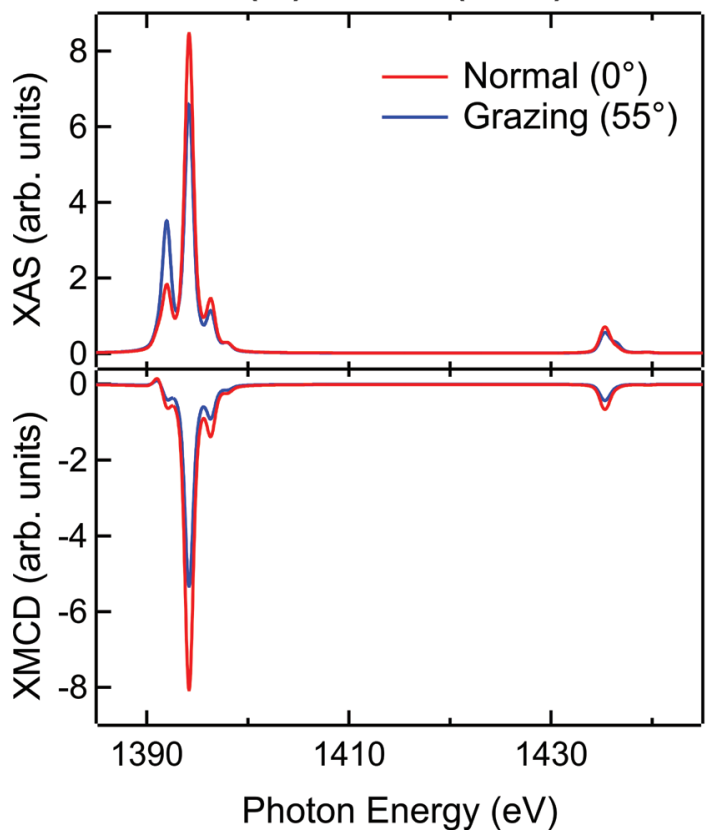

(b) $\mathrm{Er} / \mathrm{Cu}(111)$

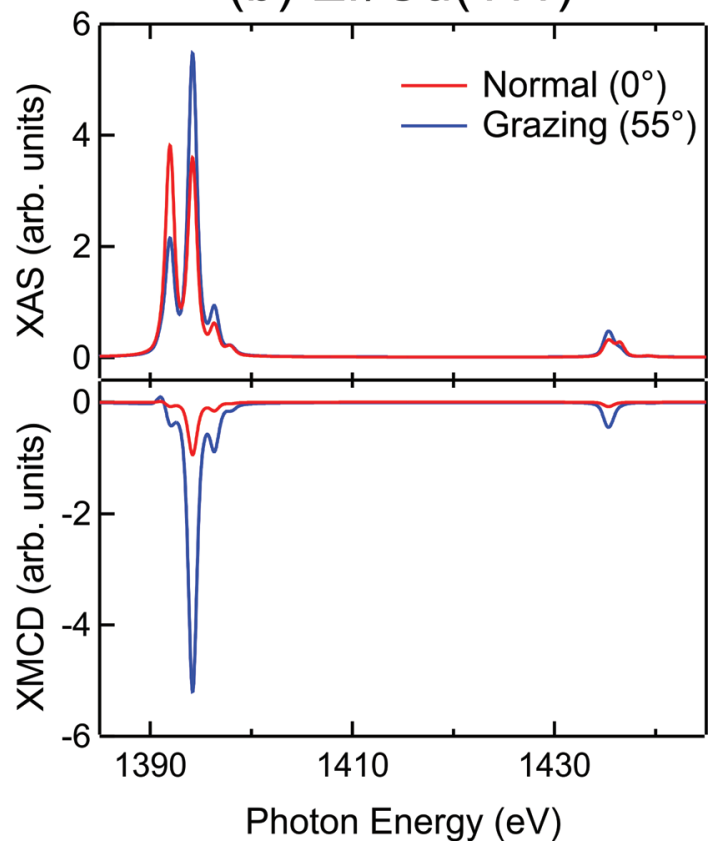

Figure S1. Simulated XAS $\left(\sigma^{+}+\sigma^{-}\right)$and XMCD $\left(\sigma^{-}-\sigma^{+}\right)$of (a) $\operatorname{Er} / \operatorname{Pt}(111)$ and (b) $\operatorname{Er} / \mathrm{Cu}(111)$ with $B=6.8 \mathrm{~T}$ and $T=2.5 \mathrm{~K}$. 
(a) $\mathrm{Ho} / \mathrm{Pt}(111)$

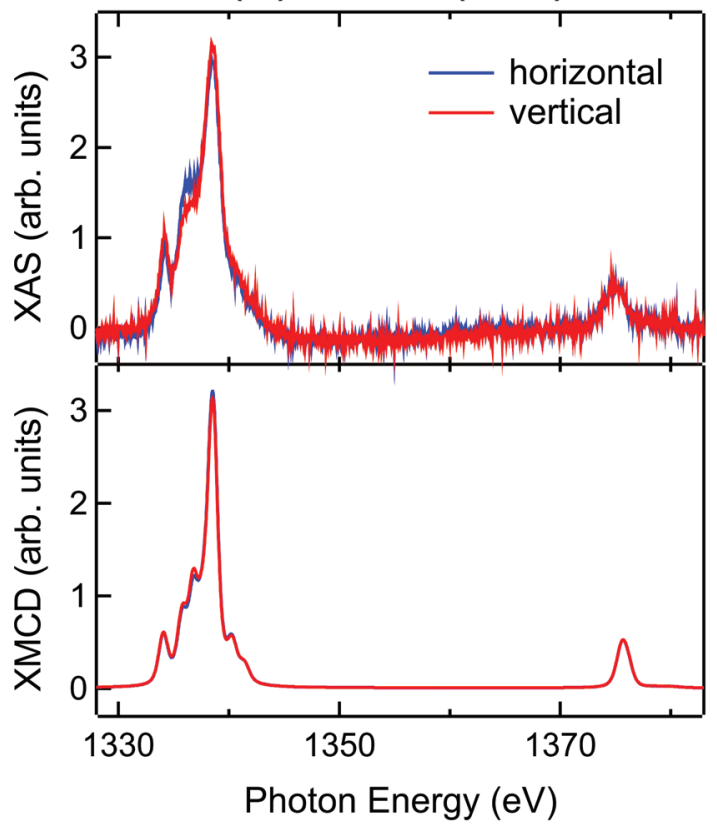

(c) $\operatorname{Er} / \mathrm{Pt}(111)$

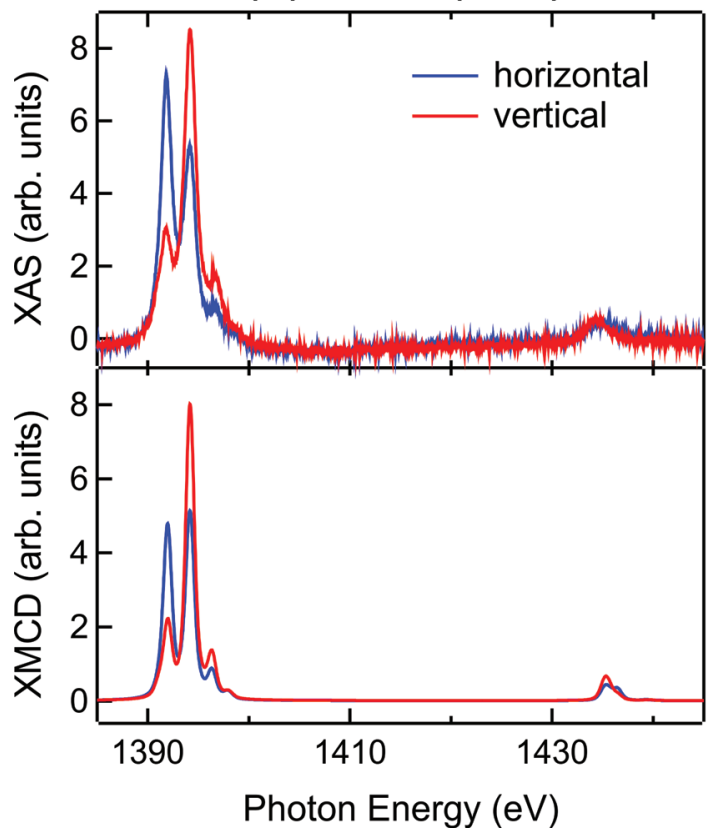

(b) $\mathrm{Ho} / \mathrm{Cu}(111)$

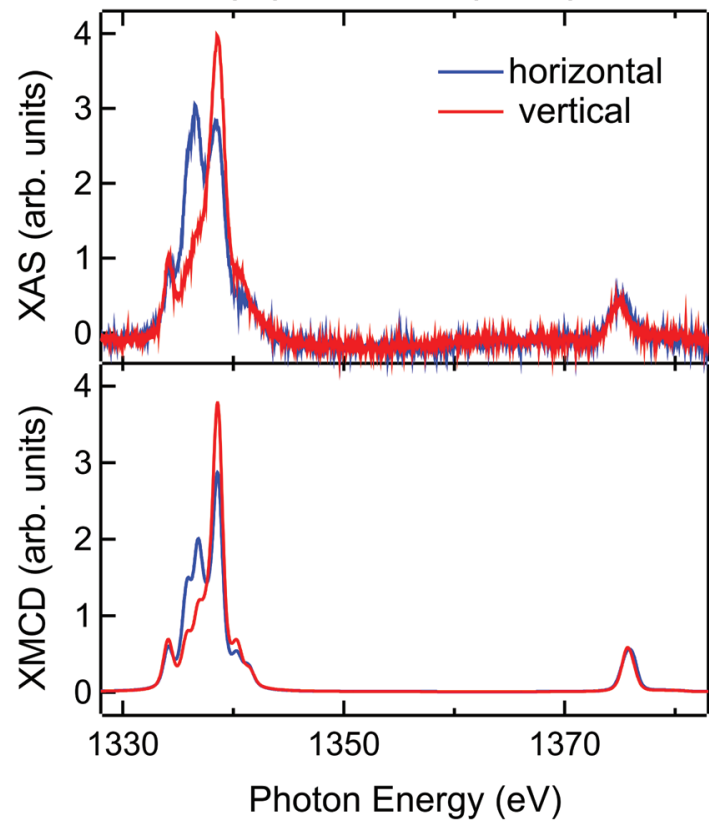

(d) $\mathrm{Er} / \mathrm{Cu}(111)$

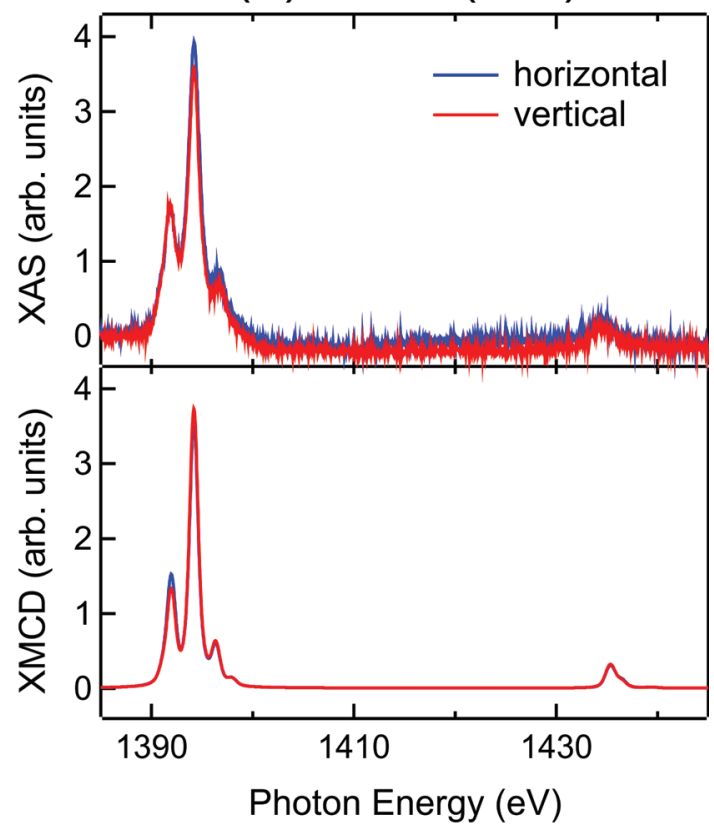

Figure S2. Experimental and simulated XAS with linear polarization obtained at grazing incidence $\left(\theta=55^{\circ}\right)$ and $B=6.8 \mathrm{~T}$, $T=2.5 \mathrm{~K}$. (a) $\mathrm{Ho} / \mathrm{Pt}(111)$, (b) $\mathrm{Ho} / \mathrm{Cu}(111)$, (c) $\mathrm{Er} / \mathrm{Pt}(111)$ and (d) $\mathrm{Er} / \mathrm{Cu}(111)$. The electric field vector is oriented parallel to (horizontal) or such to form an angle of $55^{\circ}$ (vertical) with the surface plane, while the sample is rotated by $55^{\circ}$ with respect to the beam axis. 


\section{GROUND STATE MULTIPLET FROM CALCULATIONS}

The moments and energies were calculated in a small magnetic field of $B_{z}=0.5 \mathrm{mT}$ with the $z$-axis perpendicular to the surface. Energies are given in $\mathrm{eV}$ and the ground state energy was set to zero. Note that the present values are obtained diagonalizing the full Hamiltonian acting on the complete multiplet set. Energies and moments obtained using the $B_{m}^{n}$ parameters in a crystal field Hamiltonian acting only on the ground state multiplet subset may deviate from the values presented in the tables.

\begin{tabular}{l|ccccc} 
State & $\left\langle L_{z}\right\rangle$ & $\left\langle S_{z}\right\rangle$ & $\left\langle J_{z}\right\rangle$ & $\left\langle T_{z}\right\rangle$ & $E$ \\
\hline \hline 1 & -4.5649 & -1.4351 & -6.0000 & -0.1152 & 0.000000 \\
2 & 4.5649 & 1.4351 & 6.0000 & 0.1152 & 0.000000 \\
3 & -3.8204 & -1.1796 & -5.0000 & -0.0975 & 0.003931 \\
4 & 3.8204 & 1.1796 & 5.0000 & 0.0975 & 0.003932 \\
5 & -5.3184 & -1.6816 & -7.0000 & -0.1346 & 0.005524 \\
6 & 5.3184 & 1.6816 & 7.0000 & 0.1346 & 0.005524 \\
7 & -3.0733 & -0.9267 & -4.0000 & -0.0799 & 0.012880 \\
8 & 3.0733 & 0.9267 & 4.0000 & 0.0799 & 0.012880 \\
9 & -2.3177 & -0.6823 & -3.0000 & -0.0615 & 0.023338 \\
10 & 2.3177 & 0.6823 & 3.0000 & 0.0615 & 0.023338 \\
11 & -6.0978 & -1.9022 & -8.0000 & -0.1579 & 0.025942 \\
12 & 6.0978 & 1.9022 & 8.0000 & 0.1579 & 0.025943 \\
13 & -1.5521 & -0.4479 & -2.0000 & -0.0420 & 0.032637 \\
14 & 1.5521 & 0.4479 & 2.0000 & 0.0420 & 0.032637 \\
15 & -0.7783 & -0.2217 & -1.0000 & -0.0213 & 0.038926 \\
16 & 0.7783 & 0.2217 & 1.0000 & 0.0213 & 0.038926 \\
17 & 0.0000 & -0.0000 & 0.0000 & 0.0000 & 0.041138 \\
\hline \hline
\end{tabular}

Table II. Ho/Pt(111) with axial crystal field only.

\begin{tabular}{l|ccccc} 
State & $\left\langle L_{z}\right\rangle$ & $\left\langle S_{z}\right\rangle$ & $\left\langle J_{z}\right\rangle$ & $\left\langle T_{z}\right\rangle$ & $E$ \\
\hline \hline 1 & -0.4894 & -0.1538 & -0.6433 & -0.0124 & 0.000000 \\
2 & 0.4894 & 0.1538 & 0.6432 & 0.0124 & 0.000004 \\
3 & -3.8069 & -1.1758 & -4.9826 & -0.0971 & 0.003875 \\
4 & 3.8069 & 1.1758 & 4.9826 & 0.0971 & 0.003876 \\
5 & -5.0313 & -1.5876 & -6.6189 & -0.1273 & 0.004874 \\
6 & 5.0313 & 1.5876 & 6.6189 & 0.1273 & 0.004874 \\
7 & -3.3322 & -1.0114 & -4.3435 & -0.0864 & 0.014308 \\
8 & 3.3322 & 1.0114 & 4.3436 & 0.0864 & 0.014308 \\
9 & -0.0013 & -0.0004 & -0.0017 & -0.0000 & 0.024045 \\
10 & 0.0013 & 0.0004 & 0.0017 & 0.0000 & 0.024441 \\
11 & -6.0713 & -1.8932 & -7.9645 & -0.1573 & 0.026645 \\
12 & 6.0713 & 1.8932 & 7.9645 & 0.1573 & 0.026646 \\
13 & -1.5672 & -0.4525 & -2.0197 & -0.0424 & 0.033555 \\
14 & 1.5672 & 0.4525 & 2.0197 & 0.0424 & 0.033555 \\
15 & -0.7817 & -0.2227 & -1.0044 & -0.0214 & 0.039866 \\
16 & 0.7817 & 0.2227 & 1.0044 & 0.0214 & 0.039866 \\
17 & 0.0000 & 0.0000 & 0.0000 & 0.0000 & 0.042089 \\
\hline \hline
\end{tabular}

Table III. Ho/Pt(111) including $B_{3}^{4}=5 \mu \mathrm{eV}$ (corresponding to $A_{n m}=-0.085 \mathrm{eV}$ ). 


\begin{tabular}{l|ccccc} 
State & $\left\langle L_{z}\right\rangle$ & $\left\langle S_{z}\right\rangle$ & $\left\langle J_{z}\right\rangle$ & $\left\langle T_{z}\right\rangle$ & $E$ \\
\hline \hline 1 & -5.5435 & -1.7328 & -7.2763 & -0.1426 & 0.000000 \\
2 & 5.5435 & 1.7328 & 7.2763 & 0.1426 & 0.000001 \\
3 & -4.5292 & -1.4141 & -5.9434 & -0.1166 & 0.001065 \\
4 & 4.5292 & 1.4141 & 5.9433 & 0.1166 & 0.001066 \\
5 & -0.0034 & -0.0011 & -0.0044 & -0.0001 & 0.002197 \\
6 & 0.0033 & 0.0010 & 0.0044 & 0.0001 & 0.002588 \\
7 & -3.3905 & -1.0521 & -4.4426 & -0.0878 & 0.004980 \\
8 & 3.3905 & 1.0521 & 4.4426 & 0.0878 & 0.004980 \\
9 & -2.5056 & -0.7749 & -3.2805 & -0.0651 & 0.007555 \\
10 & 2.5056 & 0.7749 & 3.2806 & 0.0651 & 0.007556 \\
11 & -0.0001 & -0.0000 & -0.0001 & -0.0000 & 0.008834 \\
12 & -0.0001 & -0.0000 & -0.0001 & -0.0000 & 0.012067 \\
13 & -1.0177 & -0.3123 & -1.3300 & -0.0266 & 0.012869 \\
14 & 1.0170 & 0.3121 & 1.3291 & 0.0266 & 0.012869 \\
15 & -0.6308 & -0.1933 & -0.8241 & -0.0165 & 0.013870 \\
16 & 0.6316 & 0.1936 & 0.8251 & 0.0166 & 0.013870 \\
17 & 0.0002 & 0.0001 & 0.0003 & 0.0000 & 0.014247 \\
\hline \hline
\end{tabular}

Table IV. Ho/Cu(111).

\begin{tabular}{l|ccccc} 
State & $\left\langle L_{z}\right\rangle$ & $\left\langle S_{z}\right\rangle$ & $\left\langle J_{z}\right\rangle$ & $\left\langle T_{z}\right\rangle$ & $E$ \\
\hline \hline 1 & -4.9890 & -1.2174 & -6.2064 & -0.2697 & 0.000000 \\
2 & 4.9890 & 1.2174 & 6.2064 & 0.2697 & 0.000000 \\
3 & -1.6331 & -0.4002 & -2.0333 & -0.0881 & 0.005318 \\
4 & 1.6330 & 0.4001 & 2.0331 & 0.0881 & 0.005318 \\
5 & -1.0834 & -0.2649 & -1.3483 & -0.0584 & 0.014118 \\
6 & 1.0835 & 0.2649 & 1.3485 & 0.0584 & 0.014118 \\
7 & -2.9464 & -0.7088 & -3.6551 & -0.1598 & 0.020632 \\
8 & 2.9464 & 0.7088 & 3.6551 & 0.1598 & 0.020632 \\
9 & -1.6005 & -0.3799 & -1.9804 & -0.0872 & 0.033257 \\
10 & 1.6006 & 0.3799 & 1.9804 & 0.0872 & 0.033257 \\
11 & -0.1234 & -0.0341 & -0.1575 & -0.0064 & 0.064928 \\
12 & 0.1231 & 0.0340 & 0.1572 & 0.0064 & 0.064928 \\
13 & -2.9206 & -0.7088 & -3.6294 & -0.1577 & 0.067023 \\
14 & 2.9206 & 0.7088 & 3.6294 & 0.1577 & 0.067023 \\
15 & -0.2577 & -0.0658 & -0.3235 & -0.0137 & 0.070545 \\
16 & 0.2580 & 0.0658 & 0.3239 & 0.0138 & 0.070545 \\
\hline \hline
\end{tabular}

Table V. Er/Pt(111). 


\begin{tabular}{l|ccccc} 
State & $\left\langle L_{z}\right\rangle$ & $\left\langle S_{z}\right\rangle$ & $\left\langle J_{z}\right\rangle$ & $\left\langle T_{z}\right\rangle$ & $E$ \\
\hline \hline 1 & -0.3819 & -0.0933 & -0.4753 & -0.0205 & 0.000000 \\
2 & 0.3819 & 0.0933 & 0.4752 & 0.0205 & 0.000000 \\
3 & -1.3394 & -0.3264 & -1.6658 & -0.0721 & 0.000746 \\
4 & 1.3394 & 0.3264 & 1.6658 & 0.0721 & 0.000746 \\
5 & -1.8675 & -0.4558 & -2.3233 & -0.1005 & 0.002192 \\
6 & 1.8675 & 0.4558 & 2.3233 & 0.1005 & 0.002192 \\
7 & -2.6505 & -0.6460 & -3.2964 & -0.1426 & 0.004358 \\
8 & 2.6505 & 0.6460 & 3.2965 & 0.1426 & 0.004358 \\
9 & -3.5022 & -0.8522 & -4.3545 & -0.1885 & 0.007017 \\
10 & 3.5022 & 0.8522 & 4.3545 & 0.1885 & 0.007018 \\
11 & -4.3335 & -1.0528 & -5.3864 & -0.2332 & 0.010172 \\
12 & 4.3335 & 1.0529 & 5.3864 & 0.2332 & 0.010173 \\
13 & -5.1811 & -1.2568 & -6.4380 & -0.2788 & 0.013793 \\
14 & 5.1811 & 1.2568 & 6.4380 & 0.2788 & 0.013794 \\
15 & -6.0214 & -1.4583 & -7.4797 & -0.3239 & 0.017990 \\
16 & 6.0214 & 1.4583 & 7.4797 & 0.3239 & 0.017991 \\
\hline \hline
\end{tabular}

Table VI. Er/Cu(111). 